\title{
Farewell Editorial from the Outgoing Editor-in-Chief
}

It is hard to believe that it has been six years since I took the helm as Editor-in-Chief of ACM Transactions on Software Engineering and Methodology (TOSEM) from David Notkin. TOSEM remains in excellent health, even in the face of many significant changes and trends-both positive and negative-that have affected its publication.

A very positive change is the journal-first initiative for software engineering, which I launched a few years ago with Matt Dwyer when he was Editor-in-Chief of IEEE Transactions on Software Engineering (TSE). Our leading software engineering conferences now include dozens of presentations from authors who choose to avail themselves of this initiative, which combines the advantages of conference presentation and networking with the advantages of journal publication, including a lack of submission deadlines, a lack of page limits (at least here at TOSEM), and iterative reviewing. Sadly, IEEE recently has chosen to institute page limits for all of its transactions (just 12 pages!). This may prove to be a boon to TOSEM, which continues to welcome authors who enjoy the ability to publish comprehensive, in-depth articles about their major research achievements. However, it also just reinforces the short-term, short-paper nature of our conferences and thus may end up negatively impacting TSE's role in journal-first publication in software engineering.

A less positive trend over the past six years is that the growing number of submissions to software engineering conferences has hijacked much of the available reviewing capacity of our community, making it ever more difficult to line up reviewers for TOSEM. This is a perpetual complaint I receive from all of my Associate Editors, and the problem is only getting worse. For instance, during my own service as Associate Editor for TOSEM, my personal record for the number of invitations I needed to send out to line up three reviewers was 12; currently, it is not uncommon for an Associate Editor to have to invite nearly two dozen people to line up just three reviewers!

Broader changes in computer science that are positively affecting TOSEM include the tremendous and widespread success now being enjoyed by artificial intelligence and machine learning, which have (finally!) entered the mainstream of computing and are revolutionizing research in every area of computer science, software engineering included.

The past six years has also seen a continued rise in open-access publication and in people vocally advocating it. While this editorial is not the place to litigate the pros and cons of open access, I will mention one aspect of the debate that I find extremely irritating, which is the habit many open access advocates have of recklessly lumping ACM together with commercial, for-profit publishers in their diatribes against controlled access. Speaking as an author and not as TOSEM Editor-inChief, I continue to believe that ACM offers the most enlightened model I am aware of for balancing the desire for open access among authors against the demands on ACM as a leading publisher of the most important scientific information in computing. That role carries a significant cost to ACM, which it judiciously manages with great generosity and without having to reward shareholders. What amazes me most of all is how little (read "not at all") so many open-access zealots avail themselves of the services that ACM provides for freely distributing ACM copyrighted material, most notably the ACM Author-Izer Service.

In terms of satisfying some of the goals I had for TOSEM at the outset of my editorship, one key goal was to increase TOSEM's breadth of coverage of software engineering. I believe we achieved

(C) 2019 Copyright held by the owner/author(s).

1049-331X/2019/02-ART1e

https://doi.org/10.1145/3301288 
this early on during my tenure, even before the rise of important new areas of software engineering, such as applications of machine learning to problems in software engineering.

One particularly gratifying milestone during my tenure was the publication last year of a thorough bibliometric study of quantity versus impact of papers in leading software engineering journals and conference proceedings [1]. The authors of that study found that TOSEM enjoys the greatest average citations per paper (ACPP) and average normalized citations per paper (AnCPP) among all venues that were studied in spite of (or possibly because of) having the lowest number of published papers among all venues. TOSEM also produced the lowest proportion of uncited papers among the venues that were studied. With a blatant lack of modesty, I feel this is strong confirmation of TOSEM's continued leadership among software engineering publications in terms of its quality and citation impact.

In closing, I would like to convey my best wishes to Mauro Pezzè, the incoming Editor-in-Chief. Mauro is an internationally leading expert in many important areas of software engineering, and he was a reliable and dedicated Associate Editor during the early part of my tenure as Editorin-Chief. I know he will do a fine job leading TOSEM in the coming years. In addition, I would like to express my sincere thanks to all of the current and past Associate Editors with whom I have worked; they have adapted to the changes and endured the difficulties mentioned above with grace and dedication. Finally, I would like to express my heartfelt thanks for the hard work of three individuals and two groups who played a critical role in maintaining TOSEM as one of the leading outlets for software engineering research, without whom I would never have survived in this job-Laura Lander, my liaison with ACM and the ACM Publications Board; Giacomo Toffetti Carughi, the TOSEM information director and webmaster; my Editorial Assistant (and wife!) Rosie Oliveira; and the communities of hardworking authors and referees who have maintained the high standards that our field expects of TOSEM.

David S. Rosenblum Editor-in-Chief (Outgoing)

\section{REFERENCE}

[1] Vahid Garousi and João M. Fernandes. 2017. Quantity versus impact of software engineering papers: A quantitative study. Scientometrics 112 (2017), 963-1006. 